ARTIGO

\title{
IMPLEMENTAÇÃO PARALELIZADA DO MÉTODO DO GRADIENTE BICONJUGADO ESTABILIZADO PARA A SIMULAÇÃO DE ESCOAMENTOS BIFÁSICOS EM RESERVATÓRIOS DE PETRÓLEO ${ }^{1}$
}

\author{
Ralph Alves Bini da Silva Almeida ${ }^{2}$ \\ Mayksoel Medeiros de Freitas \\ Grazione de Souza \\ Helio Pedro Amaral Souto
}

\begin{abstract}
RESUMO
Neste trabalho, foi implementada uma versão paralelizada do Método do Gradiente Biconjugado Estabilizado empregando-se a API OpenMP, que permitiu o uso simultâneo de threads para executar tarefas em arquiteturas com memória compartilhada, em um coprocessador Xeon Phi 3120P da Intel Corporation. Estudou-se o problema da injeção de água na recuperação de óleo em um reservatório heterogêneo com geometria do tipo Slab. O Método dos Volumes Finitos foi utilizado na discretização das equações governantes do escoamento bifásico, e o Método IMPES (Implicit Pressure Explicit Saturation) foi adotado na solução numérica das equações governantes. Os resultados numéricos mostraram que houve uma melhora da performance numérica e aumento do speedup ao refinar a malha computacional.
\end{abstract}

Palavras-chave: Método do Gradiente Biconjugado Estabilizado. OpenMP. Escoamento Bifásico. Reservatórios de Petróleo.

\section{INTRODUÇÃO}

Considera-se o escoamento imiscível bifásico, resultante da injeção de água para a produção de óleo em um reservatório, ou seja, estuda-se um caso de produção no contexto da recuperação secundária. A modelagem desse tipo de escoamento é feita empregando-se equações diferenciais parciais (EDPs) não lineares, as quais tipicamente possuem como variáveis independentes a pressão da fase óleo (não-molhante) e a saturação da fase água (molhante). Em tais problemas, a obtenção de soluções analíticas só é possível admitindo-se hipóteses simplificadoras. Assim sendo, a aplicação de metodologias numéricas para a obtenção de soluções aproximadas deve ser considerada, tendo-se como meta o estudo de casos realísticos.

\footnotetext{
1 Como citar este artigo:
}

ALMEIDA, R. A. B. S. et al. Implementação paralelizada do método Gradiente Biconjugado Estabilizado para a simulação de escoamentos bifásicos em reservatórios de petróleo. ForScience: revista científica do IFMG, Formiga, v. 7, n. 1, e00606, jan./jun. 2019. DOI: 10.29069/forscience.2019v7n1.e606.

2 Autor para correspondência: Ralph Alves Bini da Silva Almeida, ralphbini@ gmail.com 


\subsection{Simulação numérica de reservatórios}

Normalmente, um simulador computacional de reservatórios de petróleo é desenvolvido sobretudo em decorrência do desejo de engenheiros e profissionais, ligados à área de petróleo, de conhecer acuradamente o comportamento dos fluidos presentes nos reservatórios, de modo a saber em quanto tempo seria possível extraí-los e qual seria o volume total de hidrocarbonetos a ser recuperado. O método IMPES é empregado com frequência (KOU; SUN, 2004; MONTEAGUDO; FIROOZABADI, 2006) e tem como fundamento a resolução, desacoplada, das equações governantes obtidas em termos das variáveis pressão (resolvida implicitamente) e saturação (resolvida explicitamente) (COATS, 1999). Esse método apresenta um baixo custo computacional por iteração, em função da solução segregada das equações de pressão e saturação e do cálculo explícito da saturação. Diversas versões do método IMPES foram desenvolvidas e podendo-se citar, por exemplo, as contribuições de Chen, Huan e Li (2004), Kou e Sun (2004) e Watts e Shaw (2005). Contudo, todos apresentam em comum problemas de estabilidade numérica devido à determinação explícita da saturação, levando, então, a uma restrição do valor do passo de tempo que pode ser empregado (FRANC et al., 2016). Uma das formas de se melhorar a performance do método IMPES, assim como a de outras metodologias utilizadas na simulação numérica de reservatórios, é a aplicação da computação de alto desempenho (REDONDO, 2017).

\subsection{Computação de alto desempenho}

A Computação de Alto Desempenho (High Performance Computation (HPC) em inglês) consiste, de modo geral, na utilização de Unidades Gráficas de Processamento, Coprocessadores, Supercomputadores, ou Clusters de Computadores, e Softwares que permitam reduzir o tempo necessário para a execução dos programas computacionais (BACELLAR, 2010). Também enquadram-se nesta categoria as técnicas de paralelização (CHAPMAN; JOST; PAS, 2008) como, por exemplo, o uso da programação aplicando o Message Passing Interface (MPI), o Open Multi-Processing (OpenMP) ou híbridos destes (LOSADA et al., 2016).

\section{ESCOAMENTO BIFÁSICO EM RESERVATÓRIOS DE PETRÓLEO}

Quando da simulação do escoamento multifásico de fluidos, em reservatórios de petróleo, é necessário conhecer as propriedades dos fluidos, da rocha-reservatório e das interações rocha-fluido. Tais propriedades são necessárias para a resolução das equações de balanço utilizadas na modelagem do escoamento água-óleo em reservatórios de petróleo. 


\subsection{Equações de conservação para o escoamento bifásico água-óleo}

Considera-se, aqui, o escoamento bifásico isotérmico de dois fluidos imiscíveis, portanto, não há troca de massa entre as fases água e óleo (ERTEKIN; ABOU-KASSEM; KING, 2001).

\subsubsection{Equação de conservação da massa}

O princípio da conservação de massa é expresso pela equação da continuidade (ERTEKIN; ABOU-KASSEM; KING, 2001):

$$
\frac{\partial}{\partial t}\left(\phi \rho_{\alpha} S_{\alpha}\right)+\nabla \cdot\left(\rho_{\alpha} \mathbf{v}_{\alpha}\right)-\dot{q}_{m \alpha}=0
$$

onde $\phi$ é a porosidade; $\rho_{\alpha}$ é a massa específica da fase $\alpha ; S_{\alpha}$ é a saturação da fase $\alpha ; \dot{q}_{m \alpha}$ representa o termo de fonte para a fase $\alpha$ (massa por unidade de tempo por unidade de volume), e $\mathbf{v}_{\alpha}$ é a velocidade aparente da fase $\alpha$ (razão entre a vazão de escoamento e a área da seção transversal).

\subsubsection{Equação de conservação da quantidade de movimento}

Para escoamentos multifásicos, a lei de Darcy clássica deve ser modificada para levar em conta a resistência ao escoamento devido à existência de mais de uma fase (ERTEKIN; ABOU-KASSEM; KING, 2001):

$$
\mathbf{v}_{\alpha}=-\frac{k_{r \alpha}}{\mu_{\alpha}} \mathbf{k}\left(\nabla p_{\alpha}-\gamma_{\alpha} \nabla Z\right)
$$

onde $\mathbf{k}$ é o tensor de permeabilidade absoluta do meio poroso (considerado como sendo diagonal); $k_{r \alpha}$ é a permeabilidade relativa da fase $\alpha ; \mu_{\alpha}$ é a viscosidade do fluido $\alpha ; p_{\alpha}$ é a pressão da fase $\alpha ; \nabla Z$ é o gradiente de profundidade; e $\gamma_{\alpha}=\rho_{\alpha} g$, onde $g$ é a magnitude da aceleração da gravidade.

\subsection{Equações de transporte}

A partir das equações da continuidade (1) e da lei de Darcy modificada (2), pode-se obter uma equação de transporte para cada fase:

$$
\nabla \cdot\left[\frac{\rho_{\alpha} k_{r \alpha}}{\mu_{\alpha}} \mathbf{k}\left(\nabla p_{\alpha}-\gamma_{\alpha} \nabla Z\right)\right]=\frac{\partial}{\partial t}\left(\phi \rho_{\alpha} S_{\alpha}\right)-\dot{q}_{m \alpha} \quad \alpha=w, n,
$$

sendo que as formulações mais encontradas na literatura são aquelas escritas em termos da pressão da fase não molhante $(\alpha=n)$ e da saturação da fase molhante $(\alpha=w)$ (ERTEKIN; 
ABOU-KASSEM; KING, 2001; KARDALE, 2015):

$$
\nabla \cdot\left[\frac{k_{r n}}{\mu_{n} B_{n}} \mathbf{k}\left(\nabla p_{n}-\gamma_{n} \nabla Z\right)\right]=\frac{\partial}{\partial t}\left[\frac{\phi\left(1-S_{w}\right)}{B_{n}}\right]-\dot{q}_{s c n}
$$

e

$$
\nabla \cdot\left[\frac{k_{r w}}{\mu_{w} B_{w}} \mathbf{k}\left(\nabla p_{n}-\nabla p_{c}-\gamma_{w} \nabla Z\right)\right]=\frac{\partial}{\partial t}\left(\frac{\phi S_{w}}{B_{w}}\right)-\dot{q}_{s c w},
$$

onde $B=\rho_{s c_{\alpha}} / \rho_{\alpha}$ é o fator-volume-formação (FVF), $\dot{q}_{s c \alpha}=\dot{q}_{m \alpha} / \rho_{s c_{\alpha}}, p_{c}=p_{n}-p_{w}$ é a pressão capilar, e considera-se que o meio encontra-se saturado: $S_{w}+S_{n}=1$. O subscrito $s c$ indica as condições-padrão.

Para fluidos ligeiramente compressíveis, considera-se que (CRAFT; HAWKINS, 1991):

$$
B_{l}=B_{l b} \exp \left[c_{l}\left(p_{l b}-p\right)\right]
$$

com $B_{l b}$ representando o FVF do fluido no ponto de bolha; $c_{l}$, o coeficiente de compressibilidade do fluido, e $p_{l b}$, a pressão do fluido no ponto de bolha.

Segundo Petrosky e Farshad (1995), na região de óleo subsaturado, $p>p_{o b}$, a variação da viscosidade do fluido com a pressão pode ser calculada por:

$$
\mu_{o}=\mu_{o b}\left[1+c_{\mu o}\left(p-p_{o b}\right)\right]
$$

onde $c_{\mu o}$ é o coeficiente de variação da viscosidade, e $p_{o b}$ é a pressão do óleo no ponto de bolha.

Da literatura, sabe-se que a compressibilidade da rocha do reservatório (ROSA; CARVALHO; XAVIER, 2006), sendo ela pequena e constante, pode ser levada em conta por meio da relação:

$$
\phi=\phi^{0}\left[1+c_{\phi}\left(p-p^{0}\right)\right],
$$

onde $c_{\phi}$ representa o coeficiente de compressibilidade da rocha, e $\phi^{0}$ e $p^{0}$ são, respectivamente, a porosidade e pressão de referência.

Geralmente, em sistemas bifásicos, as permeabilidades relativas são funções da saturação da fase molhante. O modelo de Corey Modificado foi o escolhido para ser empregado neste trabalho (EZEKWE, 2010). Para a fase molhante, tem-se:

$$
k_{r w}\left(S_{w}\right)=k_{r w_{\max }}\left(\frac{S_{w}-S_{i w}}{1-S_{i w}-S_{n r w}}\right)^{e w}
$$

enquanto que, para a fase não-molhante:

$$
k_{r n}\left(S_{w}\right)=k_{r n_{\max }}\left(\frac{1-S_{w}-S_{n r w}}{1-S_{i w}-S_{n r w}}\right)^{e o w}
$$

sendo $S_{w}$ a saturação e $S_{i w}$ a saturação irredutível, ambas da fase molhante; e $S_{n r w}$ a saturação residual da fase não molhante. Os pontos terminais da curva, $k_{r w_{\max }}\left(S_{n r w}\right)$ e $k_{r n_{\max }}\left(S_{i w}\right)$, e os 
expoentes ew e eow são determinados através de medições feitas em laboratório ou por meio de dados experimentais obtidos para um determinado reservatório.

Empregam-se, também, leis de potência para a determinação da pressão capilar (ROSA; CARVALHO; XAVIER, 2006):

$$
p_{c}\left(s_{w}\right)=p_{c_{\max }}\left(\frac{1-S_{w}-S_{n r w}}{1-S_{i w}-S_{n r w}}\right)^{e p c}
$$

onde o ponto terminal da curva, $p_{c_{\max }}\left(S_{i w}\right)$, e o expoente epc são determinados a partir de resultados de experimentos laboratoriais ou de dados de campo.

\subsubsection{Condições inicial e de contorno}

Na busca pelas soluções das Equações (4) e (5), é necessário que sejam fornecidas as condições iniciais e de contorno associadas ao problema de escoamento. Para um instante de tempo inicial arbitrário, $t_{0}$, a condição inicial pode ser, e.g., $p\left(x, y, z, t_{0}\right)=p_{0}(x, y, z)$ e $S\left(x, y, z, t_{0}\right)=S_{0}(x, y, z)$ em todo o domínio $\Omega$, onde $p_{0}$ e $S_{0}$ representam os valores da pressão e da saturação em todo o domínio no instante inicial.

Uma condição de pressão prescrita em um poço, na face da formação, é possível e é considerada como sendo uma condição de contorno interna. Por outro lado, nas fronteiras externas, uma condição de contorno do tipo Dirichlet conduz a uma pressão prescrita na fronteira. Quando se prescreve o fluxo mássico através da fronteira do reservatório (condição do tipo Neumann) impõe-se $\rho \mathbf{v} \cdot \mathbf{n}=V(x, y, z, t)$, onde $\mathbf{n}$ é o vetor normal unitário externo à fronteira e a função $V$ fornece o valor da vazão mássica. Em fronteiras seladas (impermeáveis), não há fluxo $(V=0)$, de forma que o gradiente de pressão é nulo (ERTEKIN; ABOU-KASSEM; KING, 2001).

\section{MÉTODO DOS VOLUMES FINITOS}

No método dos volumes finitos (VERSTEEG; MALALASEKERA, 2007), os volumes de controle (ou finito) na malha são sobrepostos ao longo de todo o domínio, definindo-se os nós da malha como sendo os centros dos volumes finitos. Neles, os valores calculados das variáveis dependentes representam o valor médio da variável neste volume (ou célula). Desta forma, o primeiro passo no processo de discretização é a construção da malha computacional, efetuando-se o particionamento do domínio de resolução.

Portanto, aplica-se o processo de discretização a um domínio tridimensional, usando-se como incrementos espaciais $\Delta x$ (na direção $x$ ), $\Delta y$ (na direção $y$ ) e $\Delta z$ (na direção $z$ ). Neste caso, 
$n_{x}, n_{y}$ e $n_{z}$ volumes existem nas direções $x, y$ e $z$, respectivamente, e

$$
\sum_{i=1}^{n_{x}} \Delta x_{i}=L_{x}, \quad \sum_{j=1}^{n_{y}} \Delta y_{j}=L_{y}, \quad \sum_{k=1}^{n_{z}} \Delta z_{k}=L_{z},
$$

onde $i, j$ e $k$ são os índices associados aos volumes finitos nas direções $x, y$ e $z$, respectivamente. No caso da direção $x$, as fronteiras dos volumes são referenciadas, e.g., por $i+1 / 2, j, k$ para a interface comum aos blocos $i, j, k$ e $i+1, j, k$. Indexações análogas são utilizadas nas demais interfaces e direções do espaço. Por uma questão de simplificação, adota-se uma notação usual mais compacta, que pode ser encontrada em Versteeg e Malalasekera (2007), para a designação das faces e dos nós dos volumes finitos (Figura 1).

As equações governantes devem ser integradas no espaço, sobre um volume finito, e no tempo de $t^{n}$ a $t^{n+1}$ (VERSTEEG; MALALASEKERA, 2007). Empregando-se esquemas do tipo diferenças centradas no espaço e uma formulação conservativa para o termo transiente (AZIZ; SETTARI, 1979) obtêm-se, após feitas todas as integrações no espaço e no tempo, as formas discretizadas das Equações (4) e (5) (FREITAS, 2017):

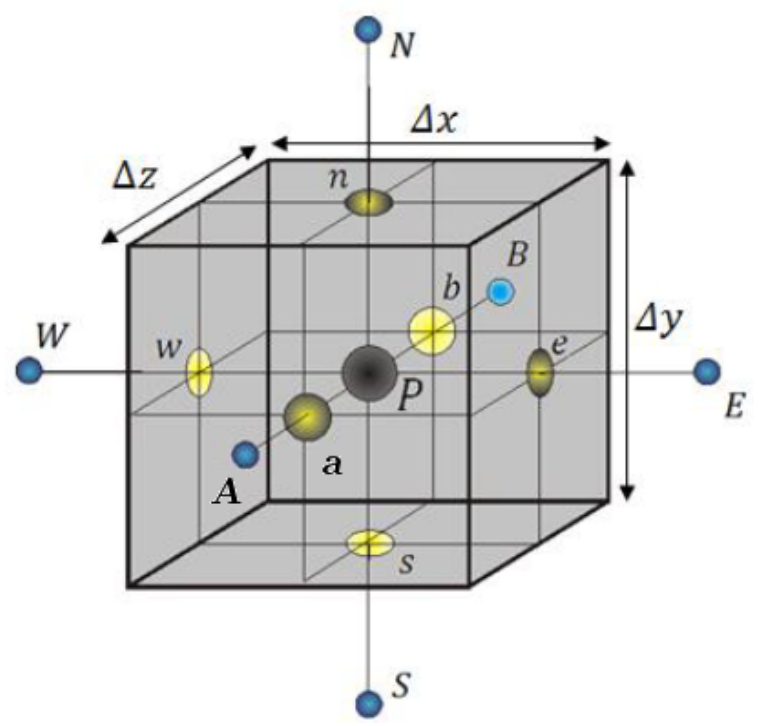

Figura 1 - Volume de integração e interfaces.

Fonte - O autor (2018)

$$
\Delta\left(T_{n} \Delta p_{n}\right)_{P}^{n+1}-\Delta\left(T_{n} \gamma_{n} \Delta Z\right)_{P}^{n+1}=C_{n p} \Delta_{t} p_{n}+C_{n s} \Delta_{t} S_{w}-q_{s c n_{P}}^{n+1}
$$

$\mathrm{e}$

$$
\Delta\left(T_{w} \Delta p_{n}\right)_{P}^{n+1}-\Delta\left(T_{w} \Delta p_{c}\right)_{P}^{n+1}-\Delta\left(T_{w} \gamma_{w} \Delta Z\right)_{P}^{n+1}=C_{w p} \Delta_{t} p_{n}+C_{w s} \Delta_{t} S_{w}-q_{s c w_{P}}^{n+1},
$$


onde

$$
\begin{gathered}
C_{n p}=\frac{V_{P}}{\Delta t}\left\{\left[\phi_{P}^{n+1}\left(\frac{1}{B_{n}}\right)_{P}^{\prime}+\frac{\phi_{P}^{\prime}}{B_{n_{P}}^{n}}\right]\left(1-S_{w_{P}}^{n}\right)\right\}, \\
C_{n s}=-\frac{V_{P}}{\Delta t}\left(\frac{\phi_{P}^{n+1}}{B_{n_{P}}^{n+1}}\right), \\
C_{w p}=\frac{V_{P}}{\Delta t}\left\{\left[\phi_{P}^{n+1}\left(\frac{1}{B_{w}}\right)_{P}^{\prime}+\frac{\phi_{P}^{\prime}}{B_{w_{P}}^{n}}\right] S_{w_{P}}^{n}\right\}, \\
C_{w s}=\frac{V_{P}}{\Delta t}\left(\frac{\phi_{P}^{n+1}}{B_{w_{P}}^{n+1}}\right),
\end{gathered}
$$

onde $\Delta_{t} \varphi=\varphi_{P}^{n+1}-\varphi_{P}^{n}$ (ABOU-KASSEM; ALI; ISLAM, 2006) e introduziu-se o operador $\Delta(\xi \Delta \eta)_{P}$, para que fosse possível reescrever as equações discretizadas numa forma compacta (ABOU-KASSEM; ALI; ISLAM, 2006),

$$
\begin{aligned}
\Delta(\xi \Delta \eta)_{P} & \equiv \xi_{x_{w}}\left(\eta_{W}-\eta_{P}\right)+\xi_{x_{e}}\left(\eta_{E}-\eta_{P}\right)+\xi_{y_{n}}\left(\eta_{N}-\eta_{P}\right)+\xi_{y_{s}}\left(\eta_{S}-\eta_{P}\right) \\
& +\xi_{z_{a}}\left(\eta_{A}-\eta_{P}\right)+\xi_{z_{b}}\left(\eta_{B}-\eta_{P}\right),
\end{aligned}
$$

com as transmissibilidades $T_{l x_{f}}$ sendo definidas, segundo Aziz e Settari (1979), por

$$
T_{l x_{f}} \equiv\left(\frac{k_{x} A_{x} k_{r l}}{\mu_{l} B_{l} \Delta x}\right)_{f} \quad l=n, w,
$$

onde $f$ é igual a $w$ ou $e$. Analogamente, podem-se obter as respectivas equações para as direções $y$ e $z$. A partir das Equações (8) e (6), para fluidos ligeiramente compressíveis, tem-se que:

$$
\left(\frac{1}{B_{l}}\right)^{\prime}=\frac{c_{l}}{B_{l}} .
$$

Assim, as Equações (13) e (14) formam um sistema de equações algébricas não lineares, que deve ser resolvido numericamente empregando-se um método apropriado.

\subsection{Implementação das condições de contorno}

Seguindo o desenvolvimento realizado por Freitas (2017), na implementação das condições de contorno, usa-se a equação geral:

$$
a_{\eta_{f}} \eta_{f}^{\prime}+b_{\eta_{f}} \eta_{f}=c_{\eta_{f}}
$$

onde $f$ designa as faces das células no contorno e $\eta^{\prime}$ a derivada espacial da variável $\eta$. Então, variando-se os valores de $a_{\eta_{f}}, b_{\eta_{f}}$ e $c_{\eta_{f}}$, é possível impor os três tipos de condições de contorno mais comuns nas engenharias: Dirichlet, Neumann ou Robin. 


\subsection{Aspectos numéricos}

Escrevem-se, agora, as transmissibilidades na forma geral (considerando a direção $x$ )

$$
T_{l x f}^{n+1}=G_{x f} F_{p l f}^{n+1} F_{S l f}^{n+1}
$$

onde

$$
G_{x f}=\left(\frac{k_{x} A_{x}}{\Delta_{x}}\right)_{f}
$$

é o termo geométrico,

$$
F_{p l f}^{n+1}=\left(\frac{1}{\mu_{l} B_{l}}\right)_{f}^{n+1}
$$

é uma função da pressão e

$$
F_{S l f}^{n+1}=\left(k_{r l}\right)_{f}^{n+1}
$$

depende da saturação, sabendo-se que elas devem ser obtidas nas interfaces $f$ dos volumes da malha computacional, sendo que as pressões e as saturações são determinadas em seus centros. Assim sendo, técnicas de interpolação devem ser utilizadas na avaliação das propriedades nas interfaces dos blocos.

Segundo Aziz e Settari (1979), para o cálculo de $F_{p}$ podem-se usar médias aritméticas. Por outro lado, na determinação de $F_{S}$, o esquema de diferenças centradas conduz a instabilidades, e métodos do tipo upwind (LEVEQUE, 2002) podem ser, alternativamente, usados para calcular $F_{S}$ nas fronteiras entre os volumes da malha (AZIZ; SETTARI, 1979).

Em geral, o termo geométrico é avaliado nas faces dos volumes finitos empregando-se uma média harmônica para o cálculo do valor da permeabilidade absoluta na interface (ERTEKIN; ABOU-KASSEM; KING, 2001).

\section{RESOLUÇÃO NUMÉRICA DO SISTEMA DE EQUAÇÕES}

O método IMPES foi desenvolvido por Sheldon, Zondek e Cardwell (1959) e Stone e Garder (1961), tendo como objetivo a obtenção da solução segregada das EDPs escritas em termos da pressão e da saturação. Para tanto, devem-se obter as pressões capilares e as transmissibilidades avaliando-as explicitamente no tempo $n$, ou no tempo $n+1$, em uma iteração conhecida $n+1, v$. Trata-se de um método eficiente e muito estudado (KOU; SUN, 2004; CHEN; HUAN; LI, 2004; MONTEAGUDO; FIROOZABADI, 2006; MEISTER; BADER, 2015; FREITAS, 2017; REDONDO, 2017). 
ALMEIDA, R. A. B. S. et al. Implementação paralelizada do método Gradiente Biconjugado Estabilizado para a simulação de escoamentos bifásicos em reservatórios de petróleo.

\subsection{Implementação do método IMPES}

A obtenção da equação para a pressão, válida em cada volume, se dá pela combinação das Equações (13) e (14) de modo que o termo $\Delta_{t} S_{w}$ seja eliminado. Assim, multiplicase a Equação (13) por $B_{n_{P}}^{n+1}$, a Equação (14) por $B_{w_{P}}^{n+1}$, adicionando ambas, sabendo-se que $B_{n_{P}}^{n+1} C_{n s}+B_{w_{P}}^{n+1} C_{w s}=0$. Então, a equação para a pressão resultante é dada por (ERTEKIN; ABOU-KASSEM; KING, 2001)

$$
\begin{aligned}
& B_{n_{P}}^{n+1} \Delta\left(T_{n} \Delta p_{n}\right)_{P}^{n+1}+B_{w_{P}}^{n+1} \Delta\left(T_{w} \Delta p_{n}\right)_{P}^{n+1}-B_{w_{P}}^{n+1} \Delta\left(T_{w} \Delta p_{c}\right)_{P}^{n+1}-B_{n_{P}}^{n+1} \Delta\left(T_{n} \gamma_{n} \Delta Z\right)_{P}^{n+1} \\
+ & B_{w_{P}}^{n+1} \Delta\left(T_{w} \gamma_{w} \Delta Z\right)_{P}^{n+1}=\left(B_{n_{P}}^{n+1} C_{n p}+B_{w_{P}}^{n+1} C_{w p}\right) \Delta_{t} p_{n}-\left(B_{n_{P}}^{n+1} q_{s c n_{P}}^{n+1}+B_{w_{P}}^{n+1} q_{s c w_{P}}^{n+1}\right),
\end{aligned}
$$

podendo ainda ser linearizada, para uma iteração de Picard (ERTEKIN; ABOU-KASSEM; KING, 2001), na forma

$$
\begin{aligned}
& -T_{t z_{a}}^{n+1, v} p_{n_{A}}^{n+1, v+1}-T_{t y_{n}}^{n+1, v} p_{n_{N}}^{n+1, v+1}-T_{t x_{w}}^{n+1, v} p_{n_{W}}^{n+1, v+1} \\
& +\left(T_{t z_{a}}^{n+1, v}+T_{t y_{n}}^{n+1, v}+T_{t x_{w}}^{n+1, v}+T_{t x_{e}}^{n+1, v}+T_{t y_{s}}^{n+1, v}+T_{t z_{b}}^{n+1, v}+C_{t p}\right) p_{n_{P}}^{n+1, v+1} \\
& -T_{t x_{e}}^{n+1, v} p_{n_{E}}^{n+1, v+1}-T_{t y_{s}}^{n+1, v} p_{n_{S}}^{n+1, v+1}-T_{t z_{b}}^{n+1, v} p_{n_{B}}^{n+1, v+1} \\
& =C_{t p} p_{n_{P}}^{n+1, v}-B_{n_{P}}^{n+1, v}\left[T_{w z_{a}}^{n+1, v}\left(p_{c_{A}}^{n+1, v}-p_{c_{P}}^{n+1, v}\right)+T_{w y_{n}}^{n+1, v}\left(p_{c_{N}}^{n+1, v}-p_{c_{P}}^{n+1, v}\right)\right. \\
& +T_{w x_{w}}^{n+1, v}\left(p_{c_{W}}^{n+1, v}-p_{c_{P}}^{n+1, v}\right)+T_{w x_{e}}^{n+1, v}\left(p_{c_{E}}^{n+1, v}-p_{c_{P}}^{n+1, v}\right)+T_{w y_{s}}^{n+1, v}\left(p_{c_{S}}^{n+1, v}-p_{c_{P}}^{n+1, v}\right) \\
& \left.+T_{w z_{b}}^{n+1, v}\left(p_{c_{B}}^{n+1, v}-p_{c_{P}}^{n+1, v}\right)\right]+q_{s c t_{P}}^{n+1, v}+\Gamma_{t_{P}}^{n+1, v},
\end{aligned}
$$

tendo sido introduzidas as seguintes variáveis:

$$
\begin{gathered}
C_{t p} \equiv B_{n_{P}}^{n+1, v} C_{n p}+B_{w_{P}}^{n+1, v} C_{w p}, \\
q_{s c t_{P}}^{n+1, v} \equiv B_{n_{P}}^{n+1} q_{s c n_{P}}^{n+1, v}+B_{w_{P}}^{n+1} q_{s c w_{P}}^{n+1, v},
\end{gathered}
$$

$\mathrm{e}$

$$
\begin{gathered}
\Gamma_{t_{P}}^{n+1, v} \equiv B_{n_{P}}^{n+1} \Gamma_{n_{P}}^{n+1, v}+B_{w_{P}}^{n+1} \Gamma_{w_{P}}^{n+1, v}, \\
q_{s c t_{P}}^{n+1, v} \equiv B_{n_{P}}^{n+1} q_{s c n_{P}}^{n+1, v}+B_{w_{P}}^{n+1} q_{s c w_{P}}^{n+1, v} .
\end{gathered}
$$

A Equação (28) deve ser resolvida de modo a determinar a pressão que é, em seguida, usada no cálculo explícito da saturação a partir da forma linearizada:

$$
\begin{aligned}
S_{w_{P}}^{n+1, v+1} & =S_{w_{P}}^{n}+\frac{1}{C_{w s}}\left[T_{w z_{a}}^{n+1, v} \Delta \Phi_{w z_{A}}^{n+1, *}+T_{w y_{n}}^{n+1, v} \Delta \Phi_{w y_{N}}^{n+1, *}+T_{w x_{w}}^{n+1, v} \Delta \Phi_{w x_{W}}^{n+1, *}\right. \\
& \left.+T_{w x_{e}}^{n+1, v} \Delta \Phi_{w x_{E}}^{n+1, *}+T_{w y_{s}}^{n+1, v} \Delta \Phi_{w y_{S}}^{n+1, *}+T_{w z_{b}, v}^{n+1, v} \Delta \Phi_{w z_{B}}^{n+1, *}-C_{w p} \Delta_{t} p_{n}+q_{s c w_{P}}^{n+1, v}\right]
\end{aligned}
$$


onde:

$$
\Delta \Phi_{w z_{A}}^{n+1, *} \equiv\left(p_{n_{A}}^{n+1, v+1}-p_{n_{P}}^{n+1, v+1}\right)-\left(p_{c_{A}}^{n+1, v}-p_{c_{P}}^{n+1, v}\right)-\gamma_{w_{a}}^{n+1, v}\left(Z_{A}-Z_{P}\right)
$$

com formas análogas podendo ser obtidas para $\Delta \Phi_{w y_{N}}^{n+1, *}, \Delta \Phi_{w x_{W}}^{n+1, *}, \Delta \Phi_{w x_{E}}^{n+1, *}, \Delta \Phi_{w y_{S}}^{n+1, *}$ e $\Delta \Phi_{w z_{B}}^{n+1, *}$.

As Equações (28) e (33) são solucionadas de forma iterativa até que a convergência seja atingida. Para se determinar o critério de interrupção das iterações define-se

$$
\theta_{p n}=\frac{\delta p_{n}^{n+1, v+1}}{p_{n}^{n+1, v+1}}
$$

e

$$
\theta_{S w}=\frac{\delta S_{w}^{n+1, v+1}}{S_{w}^{n+1, v+1}}
$$

$\operatorname{com} \delta p_{n}^{n+1, v+1}=p_{n}^{n+1, v+1}-p_{n}^{n+1, v}$ e $\delta S_{w}^{n+1, v+1}=S_{w}^{n+1, v+1}-S_{w}^{n+1, v}$ para os $1,2,3,4, \ldots N$ volumes finitos do domínio computacional. As iterações terminam quando o maior valor absoluto de $\theta_{p n}$ e $\theta_{S w}$ for menor do que o da tolerância $\left(t o l_{i t}\right)$. Uma vez que essa condição seja verificada, diz-se que a solução numérica convergiu.

$\mathrm{Na}$ prática, sabe-se que a determinação implícita da pressão exige um maior esforço computacional do que o cálculo explícito da saturação. Assim sendo, considerando-se que a pressão seja determinada a cada intervalo de tempo $\Delta t_{p}$, divide-se esse intervalo em $m$ subintervalos $\Delta t_{s}$, onde $\sum_{i=1}^{m} \Delta t_{s_{i}}=\Delta t_{p}$, que é então empregado para o cálculo da saturação (HURTADO; MALISKA; SILVA, 2006).

Durante a simulação, é interessante que se possa variar o tamanho do passo de tempo para que ele seja o maior possível a cada iteração, de maneira a reduzir o número de passos de tempo necessários para atingir o tempo máximo de simulação. Sendo $i t_{n-1}$ o número de iterações necessárias para alcançar a convergência no passo de tempo anterior $\left(\Delta t_{n-1}\right)$, pode-se adotar o seguinte critério empírico (FREITAS, 2017):

$$
\Delta t_{n}= \begin{cases}n_{\text {decr }} \Delta t_{n-1} & \text { se } \quad i t_{n-1}>i t_{\text {decr }} \\ n_{i n c r} \Delta t_{n-1} & \text { se } \quad i t_{n-1} \leq i t_{i n c r} \\ \Delta t_{n-1} & \text { caso contrário }\end{cases}
$$

onde $n_{\text {decr }}$ e $n_{\text {incr }}$ são as taxas de decrescimento e crescimento do passo de tempo; $i t_{d e c r}$ é o número mínimo de iterações para a convergência da solução, no passo de tempo anterior, no qual o passo de tempo foi reduzido; e it ${ }_{i n c r}$, o número máximo de iterações para o qual ele é incrementado ( $i t_{i n c r}<i t_{d e c r}$ ). Para tornar o critério mais conservador, o crescimento do passo de tempo é limitado a um valor máximo, $\Delta t_{n} \leq \Delta t_{\max }$. No caso da simulação não convergir para um dado passo de tempo calculado, ele é reduzido, e a simulação prosseguirá com esse novo valor do passo de tempo. 


\subsection{Solução do sistema de equações para a pressão do óleo}

A solução dos sistemas de equações algébricas lineares é, na maioria dos casos, uma das etapas que demandam o maior esforço computacional em uma simulação de reservatórios, e, geralmente, métodos iterativos são utilizados com tal objetivo (ERTEKIN; ABOU-KASSEM; KING, 2001). Dentro da classe de métodos iterativos, a alternativa escolhida para a solução do sistema linear (28) foi o Método do Gradiente Biconjugado Estabilizado (Biconjugate Gradient Stabilized Method, ou BiCGStab) (VORST, 1992).

\subsection{Processamento paralelo usando o OpenMP}

Na computação de alto desempenho, uma das técnicas conhecidas de paralelização é a Aplication Programming Interface (API) OpenMP. Em sua utilização, em arquiteturas de memória compartilhada, faz-se necessário o uso de três componentes básicos: as diretivas de compilação, a biblioteca de execução e as variáveis de ambiente (ARB, 2011). O emprego do OpenMP baseia-se no modelo de execução denominado Fork-Join, conforme ilustrado na Figura 2 (CHAPMAN; JOST; PAS, 2008). Em geral, pode-se dizer que, em algum momento, uma determinada diretiva iniciará a paralelização (thread inicial) de um trecho do código computacional, distribuindo uma sequência de tarefas entre diversas threads. Em seguida, as threads executarão as tarefas designadas de modo separado (Fork), e, posteriormente, a thread inicial reunirá todas as informações resultantes do trabalho paralelizado (Join).

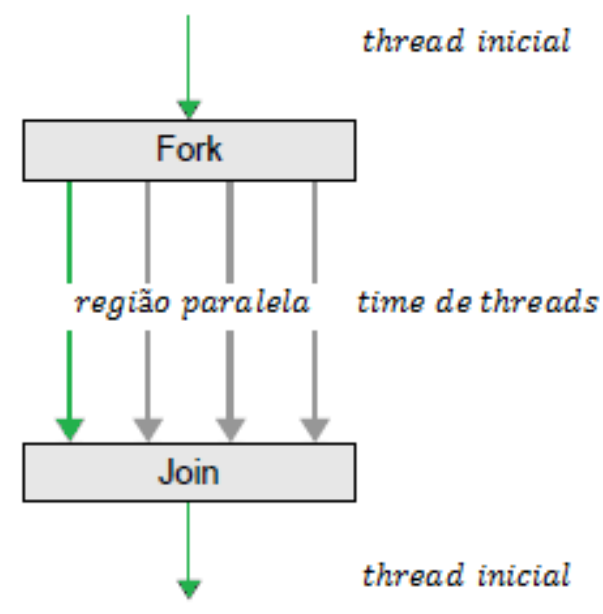

Figura 2 - Modelo Fork-Join.

Fonte - Adaptado de Werneck (2016)

Os dados utilizados nos cálculos são rotulados em dois tipos básicos de classificação: shared e private. No caso do tipo shared, existe apenas uma instância dos dados, sendo que 
ALMEIDA, R. A. B. S. et al. Implementação paralelizada do método Gradiente Biconjugado Estabilizado para a simulação de escoamentos bifásicos em reservatórios de petróleo.

todas as threads podem acessar e modificar os dados simultaneamente, a menos que alguma restrição seja imposta via comando específico do OpenMP. Todas as alterações são visíveis para todas as threads. Por outro lado, quando o tipo private é adotado, cada linha de execução possui uma cópia dos dados, que é particular. Nesse caso, as alterações são visíveis apenas para a thread que possui os dados. Uma descrição completa desses e de outros comandos pode ser encontrada em Chapman, Jost e Pas (2008).

Portanto, neste trabalho, as diretivas e os tipos de dados, \#pragma, shared e private, foram aplicados na paralelização do método BiCGSTAB. O Algoritmo 1 mostra a sequência de etapas executadas com a indicação das operações que foram paralelizadas. Observa-se, no algoritmo, que o vetor solução, $\mathbf{x}$, e o vetor residual, $\mathbf{r}$, são atualizados iterativamente.

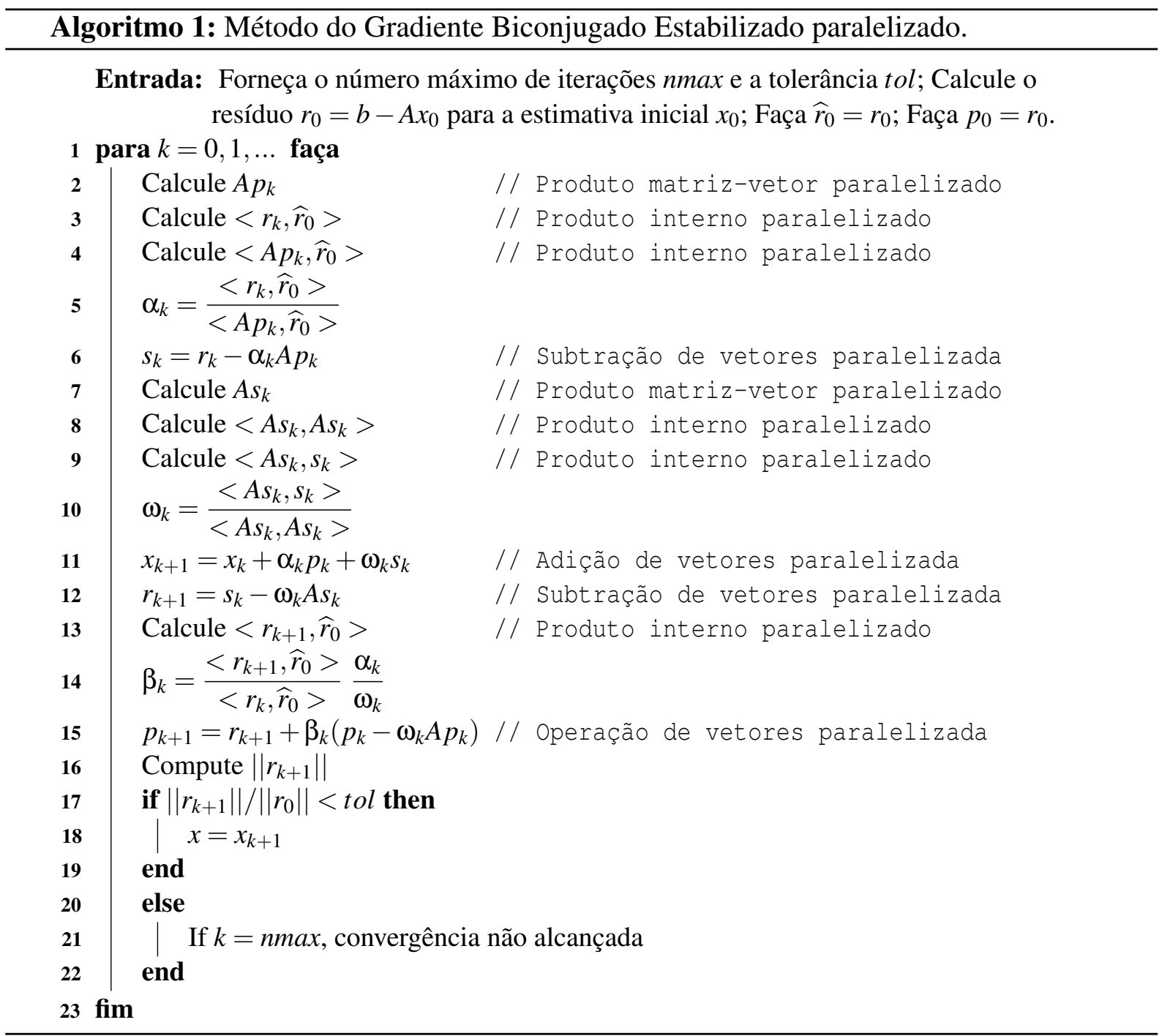




\section{RESULTADOS E DISCUSSÃO}

Nesta parte, apresentam-se os resultados obtidos com as simulações do escoamento bifásico água-óleo. O simulador foi todo desenvolvido em linguagem de programação C, e as simulações numéricas foram realizadas utilizando-se uma servidora DELL PowerEdge 730 contendo dois coprocessadores Xeon Phi 3120P, da Intel Corporation com 57 núcleos de 1,10 GHz, num total de 228 threads (4 por núcleo) e 6 GB de memória RAM cada um.

Estudou-se o problema do escoamento bifásico água-óleo em uma geometria do tipo Slab. O reservatório possui o formato de um paralelepípedo com dimensões $L_{x}, L_{y}$ e $L_{z}$ (Figura 3) e considerando-se que todas as suas fronteiras externas são impermeáveis, menos aquelas cuja normal é paralela ao eixo $x$. Na fronteira de injeção, localizada em $x=0$, impôs-se um gradiente de pressão e uma condição do tipo Dirichlet para a saturação. Por outro lado, na fronteira $x=L_{x}$, impôs-se um valor de pressão constante.

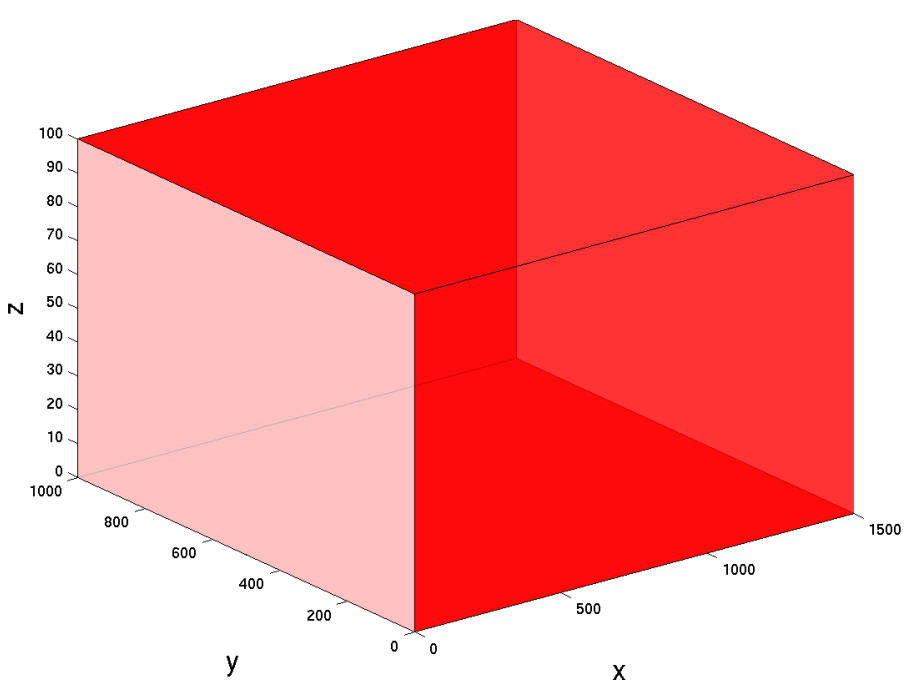

Figura 3 - Reservatório com geometria do tipo Slab.

Fonte - Adaptado de Freitas (2017)

Um caso-base (padrão) para a simulação foi construído (FREITAS, 2017). Salvo menção em contrário, esses dados foram utilizados em todas as simulações. A Tabela 1 contém as informações referentes aos fluidos (óleo e água), enquanto a Tabela 2 apresenta os parâmetros de rocha e de interação rocha-fluido. Considerou-se, como cenário de produção, a injeção de água em um reservatório contendo óleo e uma quantidade residual de água, levando a uma saturação inicial $S_{w_{0}}=0,2$. A pressão no topo do reservatório, $P_{r e f}$, é igual a 5.800 psi, sendo que, inicialmente, a pressão no reservatório é determinada de acordo com a pressão hidrostática. 
ALMEIDA, R. A. B. S. et al. Implementação paralelizada do método Gradiente Biconjugado Estabilizado para a simulação de escoamentos bifásicos em reservatórios de petróleo.

Todo o reservatório encontra-se em uma temperatura constante $T_{r e f}=150{ }^{\circ} \mathrm{F}$. Um gradiente de pressão uniforme, $\partial p / \partial x=-0,5 \mathrm{psi} / \mathrm{ft}$, no plano $y z$, foi imposto na fronteira esquerda do reservatório. O tempo total de simulação, $t_{\max }$, foi estipulado como sendo de 1.000 dias.

Tabela 1 - Propriedades dos Fluidos

\begin{tabular}{ccc|ccc}
\hline & Óleo & & \multicolumn{3}{c}{ Água } \\
\hline Parâmetro & Valor & Unidade & Parâmetro & Valor & Unidade \\
\hline$\rho_{s c_{o}}$ & 56,13 & $\mathrm{lbm} / \mathrm{ft}^{3}$ & $\rho_{s c_{w}}$ & 62,37 & $\mathrm{lbm} / \mathrm{ft}^{3}$ \\
$p_{o b}$ & 2.000 & $\mathrm{psi}$ & $p_{w b}$ & 2.000 & $\mathrm{psi}$ \\
$B_{o b}$ & 1,13 & $\mathrm{bbl} / \mathrm{STB}$ & $B_{w b}$ & 1,022 & $\mathrm{bbl} / \mathrm{STB}$ \\
$c_{o}$ & $5 \times 10^{-6}$ & $\mathrm{psi}^{-1}$ & $c_{w}$ & $18 \times 10^{-6}$ & $\mathrm{psi}^{-1}$ \\
$\mu_{o b}$ & 1,69 & $\mathrm{cp}$ & $\mu_{w b}$ & 0,69 & $\mathrm{cp}$ \\
$c_{\mu o}$ & $1,42 \times 10^{-4}$ & $\mathrm{psi}^{-1}$ & $c_{\mu w}$ & $0,52 \times 10^{-4}$ & $\mathrm{psi}^{-1}$ \\
\hline
\end{tabular}

Tabela 2 - Propriedades do Reservatório

\begin{tabular}{ccc|ccc}
\hline \multicolumn{3}{c|}{ Fluido-Rocha } & \multicolumn{3}{c}{ Rocha } \\
\hline Parâmetro & Valor & Unidade & Parâmetro & Valor & Unidade \\
\hline$S_{i w}$ & 0,15 & - & $k_{x}=k_{y}$ & 10 & $\mathrm{mD}$ \\
$S_{n r w}$ & 0,15 & - & $k_{z}$ & 7,5 & $\mathrm{mD}$ \\
$k_{r w_{\max }}$ & 0,4 & - & $\phi^{0}$ & 0,2 & - \\
$k_{r n_{\max }}$ & 0,9 & - & $c_{\phi}$ & $4 \times 10^{-6}$ & $\mathrm{psi}^{-1}$ \\
$e w$ & 4 & - & & & \\
$e o w$ & 4 & - & & & \\
$p_{c_{\max }}$ & 0 & $\mathrm{psi}$ & & & \\
$e p c$ & 2 & - & &
\end{tabular}

No estudo do desempenho computacional, escolheu-se empregar quatro malhas computacionais. As informações referentes às malhas utilizadas podem ser vistas na Tabela 3 , e o reservatório possui as dimensões: $1.500 \mathrm{ft} \times 1.000 \mathrm{ft} \times 100 \mathrm{ft}$ nas direções dos eixos $x, y \mathrm{e} z$ (Figura 3), respectivamente.

Discutem-se, em seguida, os resultados determinados para reservatórios com campos de permeabilidade absoluta heterogêneos. A heterogeneidade do campo foi gerada a partir do uso de uma função da posição (FREITAS, 2017):

$$
k(x, y)=0,001 \max [F(x, y) ; 0,3]
$$


ALMEIDA, R. A. B. S. et al. Implementação paralelizada do método Gradiente Biconjugado Estabilizado para a simulação de escoamentos bifásicos em reservatórios de petróleo.

Tabela 3 - Malhas computacionais

\begin{tabular}{crrr}
\hline Malha & $n_{x}$ & $n_{y}$ & $n_{z}$ \\
\hline 1 & 32 & 16 & 5 \\
2 & 64 & 32 & 5 \\
3 & 128 & 64 & 5 \\
4 & 256 & 128 & 5 \\
\hline \multicolumn{4}{l}{ Fonte $-O$ autor $(2018)$}
\end{tabular}

onde $k(x, y)$ representa o valor da permeabilidade absoluta na posição $(x, y)$, a função max fornece o máximo valor entre dois números reais e

$$
F(x, y)=a\left\{0,7\left[0,5 \operatorname{sen}\left(6 \pi \frac{x}{L_{x}}\right) \cos \left(12 \pi \frac{y}{L_{y}}\right)-1\right]+1\right\}
$$

com $a$ sendo chamada de amplitude da função.

Na Figura 4 encontra-se a ilustração de um campo de permeabilidade criado a partir do emprego da Equação (38) e da Malha 4. Nota-se que, apesar do campo de permeabilidade ser heterogêneo, existe um padrão que é espacialmente periódico.

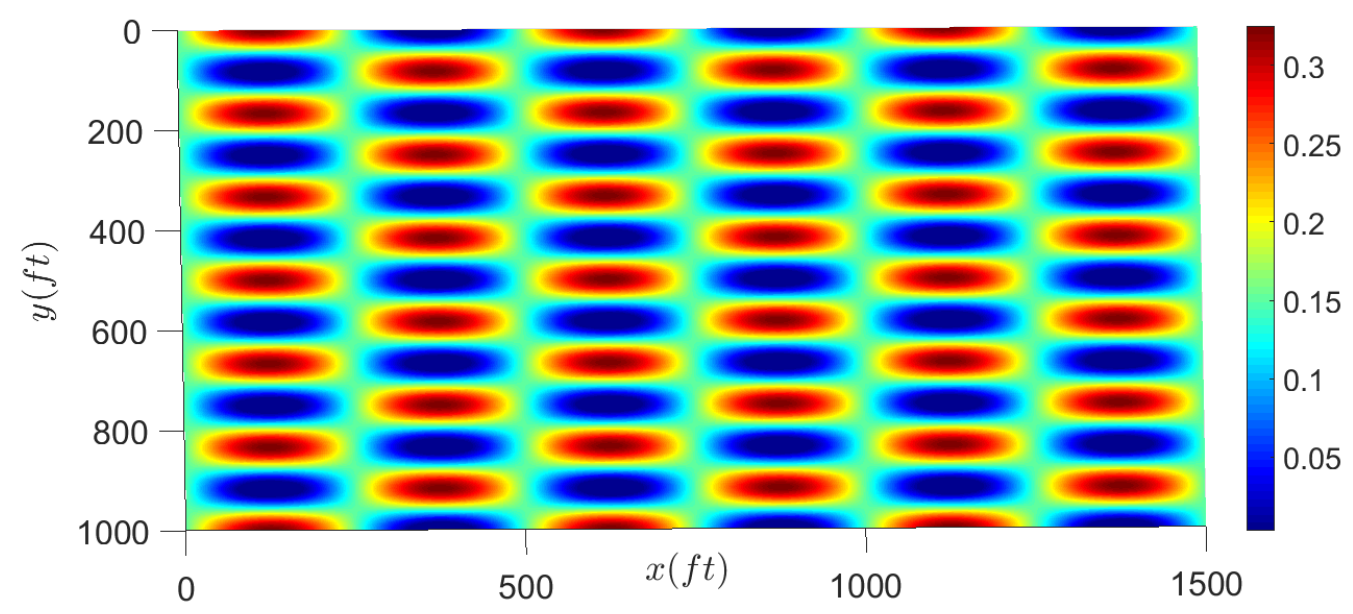

Figura 4 - Exemplo de um campo heterogêneo de permeabilidade.

Fonte - $\mathrm{O}$ autor (2018)

Nas Tabelas 4 e 5, são apresentados, respectivamente, os resultados de desempenho computacional calculados empregando-se as Malhas 1, 2, 3 e 4. Cabe ressaltar que, quando se refina a malha, o padrão de heterogeneidade também varia espacialmente, devido à Equação (38). O refinamento de malha também conduz a uma restrição no passo de tempo, como consequência do cálculo explícito da saturação, acarretando o aumento do tempo total de computação.

ForSci.: r. cient. IFMG, Formiga, v. 7, n. 1, e00606, jan./jun. 2019. 
ALMEIDA, R. A. B. S. et al. Implementação paralelizada do método Gradiente Biconjugado Estabilizado para a simulação de escoamentos bifásicos em reservatórios de petróleo.

Tabela 4 - Desempenho computacional: Malhas 1 e 2

\begin{tabular}{crr|crr}
\hline & Malha 1 & \multicolumn{3}{|c}{ Malha 2 } \\
\hline Threads & $t_{\text {OpenMP }} \mathbf{S}$ & Speedup & Threads & $t_{\text {OpenMP }} \mathbf{S}$ & Speedup \\
\hline 1 & 831 & - & 1 & 4.725 & - \\
20 & 866 & 0,96 & 20 & 2.842 & 1,66 \\
40 & 937 & 0,99 & 40 & 3.026 & 1,56 \\
60 & 1.108 & 0,75 & 60 & 3.303 & 1,43 \\
80 & 1.096 & 0,76 & 80 & 3.437 & 1,37 \\
100 & 1.107 & 0,75 & 100 & 3.371 & 1,40 \\
120 & 1.157 & 0,72 & 120 & 3.632 & 1,30 \\
\hline \multicolumn{5}{c}{ Fonte - O autor (2018) }
\end{tabular}

Tabela 5 - Desempenho computacional: Malhas 3 e 4

\begin{tabular}{crr|crr}
\hline \multicolumn{3}{c|}{ Malha 3 } & \multicolumn{3}{c}{ Malha 4 } \\
\hline Threads & $t_{\text {OpenMP }} \mathbf{S}$ & Speedup & Threads & $t_{\text {OpenMP }} \mathbf{S}$ & Speedup \\
\hline 1 & 35.437 & - & 1 & 169.297 & - \\
20 & 12.949 & 2,74 & 20 & 65.777 & 2,57 \\
40 & 12.267 & 2,89 & 40 & 62.559 & 2,71 \\
60 & 13.166 & 2,70 & 60 & 64.687 & 2,62 \\
80 & 13.211 & 2,68 & 80 & 64.500 & 2,62 \\
100 & 13.509 & 2,62 & 100 & 63.950 & 2,65 \\
120 & 14.375 & 2,47 & 120 & 66.762 & 2,54 \\
\hline \multicolumn{5}{c}{ Fonte - O autor (2018) }
\end{tabular}

Quando se leva em consideração todas as malhas, o melhor desempenho foi alcançado com a Malha 3 (40.960 volumes finitos), com um total de 40 threads. Nesse caso, o speedup atingiu o valor de 2,89. Por outro lado, para as simulações com a Malha 4, a mais refinada, com 163.840 volumes finitos, um ganho de desempenho não desprezível também foi obtido com a paralelização do método BiCGSTAB. O melhor resultado foi atingido com 40 threads e um speedup igual a 2,71. Maiores valores para o speedup poderiam ser obtidos com o uso de técnicas de compressão de matrizes (WERNECK, 2016), mas o presente trabalho não contemplou o seu emprego e tratou somente das formas tradicionais de armazenamento de matrizes.

De fato, o número ideal de threads, provavelmente, pode não estar contemplado com os valores apresentados nas tabelas. Talvez, para um dado problema, o valor ideal possa ser, e.g., 30 threads para a Malha 3 e 50 threads para a Malha 4. De qualquer maneira, o conhecimento exato desse valor não influencia no comportamento geral e a tendência de ganho de performance, descritos a partir da análise dos resultados obtidos com a realização das simulações computacionais aqui apresentadas. 
ALMEIDA, R. A. B. S. et al. Implementação paralelizada do método Gradiente Biconjugado Estabilizado para a simulação de escoamentos bifásicos em reservatórios de petróleo.

Finalizando, a Figura 5 apresenta o avanço da frente de saturação da água no reservatório heterogêneo, para a camada central do meio poroso em relação ao eixo $z$, utilizando-se a malha mais refinada (Malha 4). Nota-se a presença de caminhos preferenciais para o escoamento do fluido, o que era esperado, devido à existência de regiões com maiores valores da permeabilidade absoluta, que oferecem uma menor resistência ao deslocamento dos fluidos.

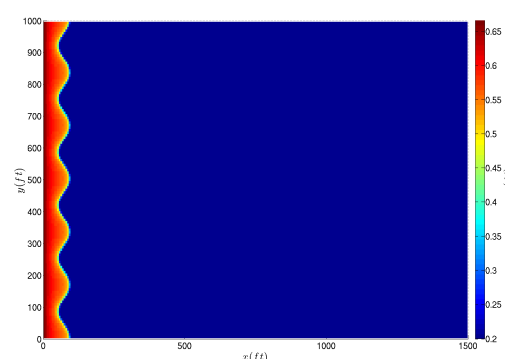

(a) 200 dias

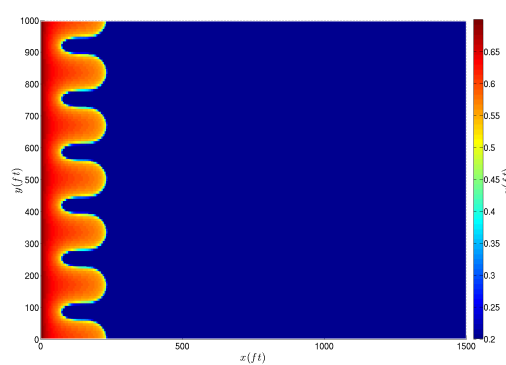

(d) 500 dias

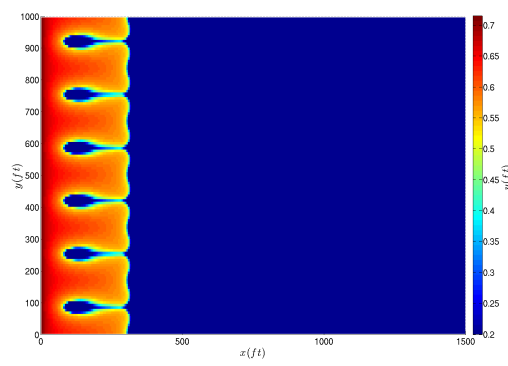

(g) 800 dias

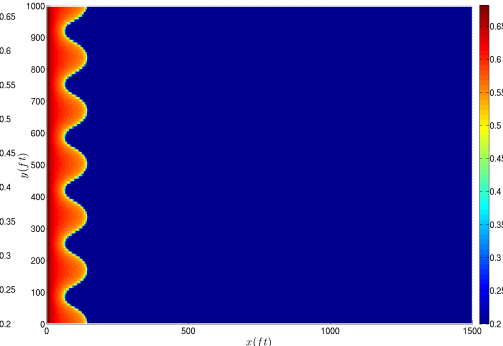

(b) 300 dias

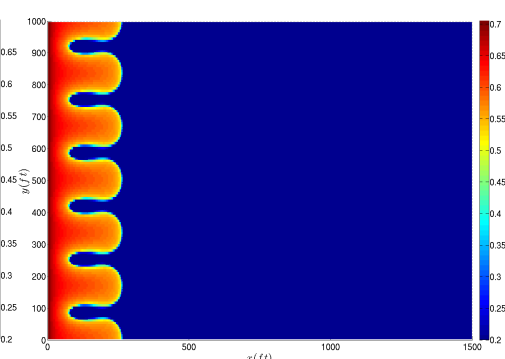

(e) 600 dias

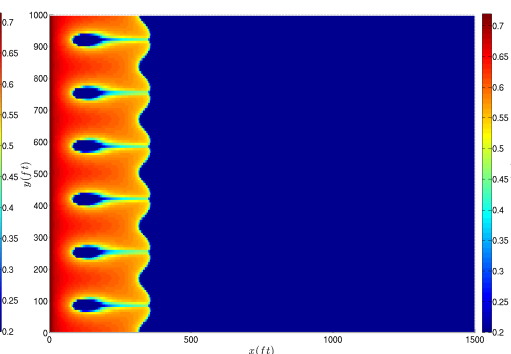

(h) 900 dias

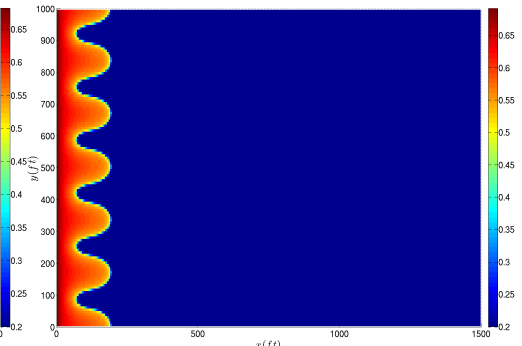

(c) 400 dias

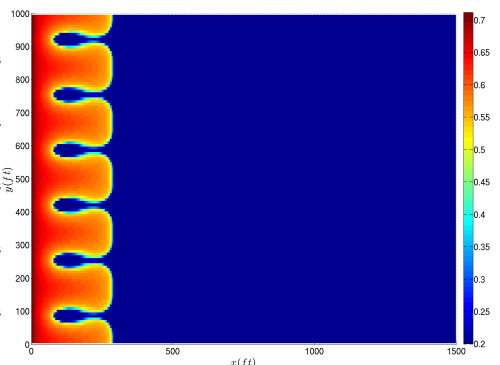

(f) 700 dias

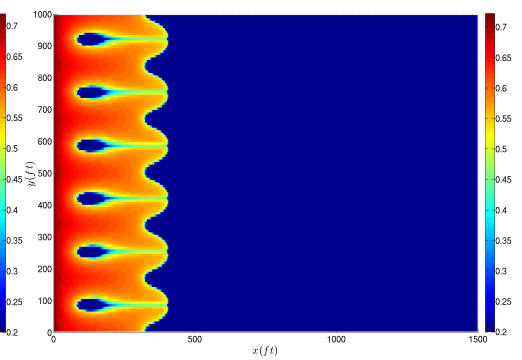

(i) 1.000 dias

Figura 5 - Avanço da frente de saturação da água no meio heterogêneo.

Fonte - O autor (2018)

\section{CONCLUSÕES}

Ganhos de performance computacional foram obtidos considerando-se o uso do método IMPES, da paralelização do método BiGSTAB, com a API OpenMP, e do emprego do coprocessador Intel Xeon Phi 3120P. Observou-se uma tendência geral de ganho de speedup com 
o aumento do número de volumes finitos da malha computacional, sendo que, para a malha menos refinada, a paralelização não conseguiu compensar o tempo de execução despendido no processo do tipo Fork-Join da API OpenMP associado ao uso das threads. Uma tendência de estagnação também foi observada à medida que o número de threads era incrementado, o que vai de encontro aos casos reportados na literatura (WERNECK, 2016). Como alternativa, uma programação mais avançada utilizando o OpenMP e o uso de técnicas de compressão de matrizes deveriam ser preconizados para que maiores ganhos sejam possíveis.

\title{
AGRADECIMENTOS
}

Os autores gostariam de agradecer o suporte financeiro fornecido pelo CNPq e pela FAPERJ.

\section{PARALLELIZED IMPLEMENTATION OF THE BICONJUGATE GRADIENT STABILIZED METHOD FOR THE SIMULATION OF TWO-PHASE FLOW IN OIL RESERVOIRS}

\begin{abstract}
In this work, we have implemented a parallelized version of the Biconjugate Gradient Stabilized Method using the OpenMP API, which allowed simultaneous use of threads to execute tasks, in shared memory architectures, in a coprocessor Intel Xeon Phi 3120P. We have also studied the problem of water injection in oil recovery in a heterogeneous reservoir with slab type geometry. We have used the Finite Volumes Method in the discretization of the governing equations of the two-phase flow and adopted the IMPES (Implicit Pressure Explicit Saturation) Method in the numerical solution of the governing equations. The numerical results have shown that there was an improvement in computational performance and increase of speedup while refining the mesh.
\end{abstract}

Keywords: Biconjugate Gradient Stabilized Method. OpenMP. Two-Phase Flow. Oil Reservoirs.

\section{REFERÊNCIAS}

ABOU-KASSEM, J. H.; ALI, S. M.; ISLAM, M. R. Petroleum Reservoir Simulation, A Basic Approach. Houston,USA: Gulf Publishing Company, 2006.

ARB. OpenMP application program interface. Version 3.1. [S.1.], 2011.

AZIZ, M.; SETTARI, A. Petroleum Reservoir Simulation. New York, USA: Elsevier Applied Science, 1979. 
BACELLAR, H. Cluster: Computação de alto desempenho. Universidade de Campinas, 2010.

CHAPMAN, B.; JOST, G.; PAS, R. van der. Using OpenMP: Portable Shared Memory Parallel Programming. Cambridge, USA: Massachusetts Institute of Technology, 2008.

CHEN, Z.; HUAN, G.; LI, B. An improved IMPES method for two-phase flow in porous media. Transport in Porous Media, v. 54, n. 3, p. 361-376, 2004.

COATS, K. H. A note on IMPES and some IMPES-based simulation models. In: 15th Symposium on Reservoir Simulation. Houston, Texas, USA: [s.n.], 1999. SPE 49774.

CRAFT, B.; HAWKINS, M. Applied petroleum reservoir engineering. [S.1.]: Englewood Cliffs, NJ.. Prentice Hall,, 1991.

ERTEKIN, T.; ABOU-KASSEM, J.; KING, G. Basic Applied Reservoir Simulation. Richardson, USA: Society of Petroleum Engineers, 2001.

EZEKWE, N. Petroleum Reservoir Engineering Practice. Westford, USA: Prentice Hall, 2010.

FRANC, J. et al. Benchmark of different CFL conditions for IMPES. Comptes Rendus Mécanique, n. 344, p. 715-724, 2016.

FREITAS, M. M. Estudo Comparativo das Estratégias de Solução Numérica para Escoamentos Bifásicos em Reservatórios de Petróleo. 2017. Tese (Doutorado) Universidade do Estado do Rio de Janeiro, 2017.

HURTADO, F. S. V.; MALISKA, C. R.; SILVA, A. F. C. A variable timestep strategy for accelerating the IMPES solution algorithm in reservoir simulation. In: Proceeding of the XXVII Iberian Latin American Congress on Computational Methods in Engineering. Belém, Pará, Brasil: [s.n.], 2006.

KARDALE, M. Efficient and accurate simulation of nonlinearly coupled multiphase flow in porous media. 2015. Dissertação (Mestrado) - Delft University of Technology, Delft, Netherlands, 2015.

KOU, J.; SUN, S. On iterative IMPES formulation for two-phase flow with capillarity in heterogeneous porous media. International Journal of Numerical Analysis and Modeling, v. 1, n. 1, p. 20-40, 2004.

LEVEQUE, R. J. Finite Volume Methods for Hyperbolic Problems. United Kingdon: Cambridge University Press, 2002.

LOSADA, N. et al. Portable application-level checkpointing for hybrid MPI-OpenMP applications. Procedia Computer Science, v. 80, p. 19-29, 2016.

MEISTER, O.; BADER, M. 2D adaptivity for 3D problems: Parallel SPE 10 reservoir simulation on dynamically adaptive prism grids. Journal of Computational Science, v. 9, p. 101-106, 2015. 
MONTEAGUDO, J. E. P.; FIROOZABADI, A. Comparison of fully implicit and IMPES formulations for simulation of water injection in fractured and unfractured media.

International Journal for Numerical Methods in Engineering, n. 69, p. 698-728, 2006.

PETROSKY, G. E.; FARSHAD, F. F. Viscosity correlation for the Gulf of Mexico oils. In: Society of Petroleum Engineers Production Operations Symposium, 02-04 April, SPE 29468. Oklahoma City, Oklahoma, USA: [s.n.], 1995.

REDONDO, C. A Fast IMPES Multiphase Flow Solver in Porous Media for Reservoir Simulation. 2017. Tese (Doutorado) - Universidad Politécnica de Madrid - Escuela Técnica Superior de Ingenieros Aeronáuticos, 2017.

ROSA, A. J.; CARVALHO, R. S.; XAVIER, J. A. D. Engenharia de Reservatórios de Petróleo. Rio de Janeiro, Brasil: Interciência, 2006.

SHELDON, J. W.; ZONDEK, B.; CARDWELL, W. T. One dimensional, incompressible, noncapillary, two-phase fluid flow in a porous medium. AIME, v. 1, n. 216, p. 290-296, 1959.

STONE, H. L.; GARDER, A. O. Analysis of gas-cap or dissolved-gas reservoir. AIME, v. 1, n. 222, p. 94-104, 1961.

VERSTEEG, H. K.; MALALASEKERA, W. An introduction to Computational Fluid Dynamics: the finite volume method. [S.1.]: Pearson, 2007.

VORST, H. A. D. BiCGSTAB: a fast and smoothly converging variant of BiCG for the solution of nonsimetric linear systems. SIAM Journal on Scientific and Statistical Computing, v. 13, n. 2, p. 631-644, 1992.

WATTS, J. W.; SHAW, J. S. A new method for solving the implicit reservoir simulation matrix equation. In: SPE Reservoir Simulation Symposium. Houston, Texas, USA: [s.n.], 2005. SPE 93068.

WERNECK, L. Implementação Paralelizada de Métodos de Resolução de Sistemas Algébricos na Simulação de Reservatórios de Gás. 2016. Dissertação (Mestrado) Universidade do Estado do Rio de Janeiro, Nova Friburgo, 2016. 


\section{DADOS DOS AUTORES}

\section{Ralph Alves Bini da Silva Almeida}

E-mail: ralphbini@gmail.com

Currículo Lattes: http://lattes.cnpq.br/7709894774591511

Ralph Alves Bini da Silva Almeida é Engenheiro Mecânico e concluiu sua graduação no Instituto Politécnico do Rio de Janeiro (IPRJ), Campus regional da Universidade do Estado do Rio de Janeiro (UERJ). Durante a Graduação adquiriu experiência profissional ao estagiar remuneradamente pela empresa Águas de Nova Friburgo Ltda, atuando no setor de manutenção eletromecânica e permanecendo na empresa por dois anos. Na área de Métodos Numéricos realizou seu Projeto de Graduação, tratando do escoamento bifásico água-óleo em meios porosos.

\section{Mayksoel Medeiros de Freitas}

E-mail: maykmedeiros@yahoo.com.br

Currículo Lattes: http://lattes.cnpq.br/3199715225291891

Possui Doutorado em Modelagem Computacional pelo Instituto Politécnico da Universidade do Estado do Rio de Janeiro (2017), Mestrado em Modelagem Computacional pelo Instituto Politécnico da Universidade do Estado do Rio de Janeiro (2013) e Graduação em Engenharia Mecânica pelo Instituto Politécnico da Universidade do Estado do Rio de Janeiro (2011). Tem experiência na área de Modelagem Computacional, com ênfase em Fenômenos de Transporte, atuando principalmente nos seguintes temas: dinâmica dos fluidos, escoamentos monofásicos e multifásicos em meios porosos utilizando o método dos volumes finitos, método de diferenças finitas e o método Smoothed Particle Hydrodynamics (SPH), e no desenvolvimento científico de códigos computacionais nas linguangens $\mathrm{C}$ e Python. Profissionalmente, já atuou como professor substituto na Universidade do Estado do Rio de Janeiro nos cursos de Engenharia Mecânica e de Computação e como professor na Universidade Candido Mendes nos cursos de Engenharia Civil e de Produção. Atualmente é docente na Universidade Federal do Oeste da Bahia (UFOB), regime de $40 \mathrm{~h}$ com dedicação exclusiva.

\section{Grazione de Souza}

E-mail: grazione@gmail.com

Lattes: http://lattes.cnpq.br/7987813860992687

Grazione de Souza é Engenheiro Mecânico, com ênfase em Termofluidodinâmica. Durante a Graduação teve duas experiências de Iniciação Científica. A primeira na área de Química dos Materiais, com foco em Materiais Cerâmicos. A segunda, na área de Métodos Numéricos, tendo estudado métodos para problemas de transporte convectivo não-linear. $\mathrm{Na}$ área de Métodos $\mathrm{Nu}$ méricos também realizou seu Projeto de Graduação, tratando do escoamento bifásico água-óleo em meios porosos. Também é Mestre em Modelagem Computacional na linha de pesquisa de Matemática Aplicada e Computação Científica, sendo o foco da Dissertação a simulação de escoamentos bifásicos água-óleo em reservatórios heterogêneos portadores de hidrocarbonetos. Concluiu Doutorado em Engenharia de Reservatórios e Exploração, na linha de pesquisa de Engenharia de Reservatórios, na área de Simulação Numérica, com foco na modelagem do escoamento acoplado poço-reservatório em reservatórios portadores de gás natural. Em seguida, 
realizou estágio de Pós-doutorado no tema de simulação de escoamentos em reservatórios naturalmente fraturados portadores de gás natural. Atualmente, Grazione de Souza é Professor Adjunto da Universidade do Estado do Rio de Janeiro, atuando na Área de Matemática Aplicada. Também é Advisor Professor do Student Chapter da Society of Petroleum Engineers na UERJ.

\section{Helio Pedro Amaral Souto}

E-mail: helio@iprj.uerj.br

Lattes: http://lattes.cnpq.br/1999072996132654

Possui graduação em Engenharia Mecânica pela Universidade Federal Fluminense (1983), Diplôme d'Etudes Approfondies (Engenharia Mecânica) - Institut National Polytechnique de Lorraine (1989), mestrado em Engenharia Mecânica pela Pontifícia Universidade Católica do Rio de Janeiro (1987) e doutorado em Mécanique et Energétique - Institut National Polytechnique de Lorraine (1993). Atualmente é Professor Titular da Universidade do Estado do Rio de Janeiro. Tem experiência na área de Engenharia Mecânica, com ênfase em Fenômenos de Transporte, atuando principalmente nos seguintes temas: escoamentos multifásicos em meios porosos, transporte de contaminantes em meios porosos e método dos volumes finitos para leis de conservação. 